\title{
Potencial de produção de biodiesel com espécies oleaginosas nativas de Rondônia, Brasil
}

\author{
Rosalvo STACHIW ${ }^{1 *}$, Sylviane Beck RIBEIRO ${ }^{1}$, Mário Augusto Gonçalves JARDIM², Dalvan \\ POSSIMOSER 3 , Wesley da Cunha ALVES ${ }^{3}$, Wanderson Cleiton Schmidt CAVALHEIRO3 \\ 1 Universidade Federal de Rondônia, Programa de Pós-graduação em Ciências Ambientais, Av. Norte Sul, 7.300, Nova Morada, 76940-000, Rolim de Moura, Rondônia, Brasil. \\ 2 Museu Paraense Emílio Goeldi, Coordenação de Botânica, Av. Magalhães Barata, 376, 66040-170, Belém, Pará, Brasil. \\ 3 Universidade Federal de Rondônia, Departamento de Engenharia Florestal, Av. Norte Sul, 7.300, Nova Morada, 76940-000, Rolim de Moura, Rondônia, Brasil. \\ * Autor correspondente: rosalvo_stachiw@yahoo.com.br
}

\section{RESUMO}

O plano de utilização sustentável de Produtos Florestais, especialmente as espécies oleaginosas nativas tem como função a manutençáo da floresta, a preservação do meio ambiente e a geração de renda em comunidades rurais. Neste sentido, este trabalho teve como objetivo avaliar a potencialidade de produção de biodiesel a partir de espécies oleaginosas nativas do estado de Rondônia. Para a identificação das espécies oleaginosas foram selecionadas cinco unidades amostrais em áreas com mata primária com o auxílio do método da Área Fixa. A extração do óleo das espécies oleaginosas foi realizada e aquelas com maior teor de lipídeos foram usadas para a produção de biodiesel. Os resultados mostraram que as espécies andiroba (Carapa guianensis), babaçu (Orbignya phalerata), castanha do Brasil (Bertholletia excelsa), tucumã-do-Amazonas (Astrocaryum tucuma) possuem potencial de produção de biodiesel. Especialmente o tucumã-do-Amazonas, por seu alto teor de lipídios (21,5\%) e a qualidade do biodiesel produzido. Além disso, já havia um comércio para esta oleaginosa na regiáo. Entretanto, torna-se imprescindível o estudo da cadeia produtiva destas espécies como estratégia para minimizaçáo na perda da cobertura vegetal nativa e na manutenção da função ambiental das áreas a serem exploradas.

PALAVRA-Chave: Florística, Palmeiras, Sustentabilidade, Inclusão Social.

\section{Potential of biodiesel production with oil seed native species from Rondônia, Brazil}

\begin{abstract}
The plan for sustainable use of Forest Products, especially the native oil seed species, has the function of maintaining the forest, environmental conservation and income production in rural communities. Thus, this paper aimed to evaluate the potential production of biodiesel from native oil seed species. For the identification, five oil seed species sampling units were selected in areas with primary forest using the Fixed Area method. The extraction of oil from oil seed species was carried out and those with higher levels of lipids were used for the production of biodiesel. The results showed that species andiroba (Carapa guianensis), babassu (Orbignya phalerata), Brasil nut (Bertholletia excelsa), "tucumã-do-Amazonas" (Astrocaryum tucuma) all of them have potential for biodiesel production. Especially "tucumã-do-Amazonas" for its high level of lipids (21.5\%) and the quality of the produced biodiesel. In addition, there was already a trade for this oil seed in the region. However, it becomes essential to study the productive chain of these species as a strategy to minimize the loss of native vegetation and maintaining the environmental function of the areas to be explored.
\end{abstract}

KEYWORDS: Floristic, Palm Trees, Sustainability, Social Inclusion. 


\section{INTRODUÇÃO}

O Programa Nacional de Produção e Uso de Biodiesel (PNPB) criado por Decreto Lei em 23 de dezembro de 2003 e complementado pela Lei no 11.097 de 13 de janeiro de 2005, estabelece que parte do biodiesel necessite seja produzido por cooperativas ou associaçôes de pequenos agricultores instaladas nas Regióes Norte, Nordeste e Centro-Oeste. Isto porque, as diretrizes do PNPB orientam à implantação de um programa sustentável que promova a inclusão social, no sentido de apoiar a agricultura familiar.

Em consonância ao PNPB, o Programa de desenvolvimento sustentável da Amazônia, promovido pelo governo federal, alerta que uma das formas de combater a destruiçáo dos recursos naturais e melhorar a qualidade de vida da populaçáo vivente na Floresta Amazônica é a utilização dos produtos da floresta. Neste contexto, as plantas oleaginosas nativas podem constituir a base de um modelo de desenvolvimento tecnológico e industrial autossustentado (Barbosa et al. 2009).

Além disso, as oleaginosas podem ser utilizadas para a consolidação de programas de energia renovável (Villela et al. 2014) para a produção de biodiesel, pois proporcionam apoio à agricultura familiar, criando melhores condiçôes de vida (infraestrutura) em regióes carentes e valorizando potencialidades regionais. Esta alternativa ajuda na resolução de problemas econômicos e socioambientais de difícil solução, como é o caso da sustentabilidade Amazônica (Ramos et al. 2003; Ramos e Wilhelm 2005; Iha et al. 2014). Em Rondônia, algumas oleaginosas podem ser apontadas como precursoras deste sistema: babaçu (Orbignya barbosiana Burret), castanha do Brasil (Bertholletia excelsa Bonpl.), andiroba (Carapa guianensis Aubl.), amendoim (Arachis hypogaea L.) e tucumã (Astrocaryum tucuma Mart.).

No contexto da Amazônia legal, Rolim de Moura e os municípios circunvizinhos assumem importância singular, pois possuem remanescentes de florestas com espécies oleaginosas nativas que podem contribuir na produção de biodiesel, sob o aspecto de indicadores de sustentabilidade. Neste sentido, este trabalho teve como objetivo avaliar o potencial de produção de biodiesel a partir de oleaginosas nativas da região de Rolim de Moura-RO.

\section{MATERIAL E MÉTODOS}

\section{Definição da área de estudo}

A seleçáo do espaço amostral da regiáo de Rolim de Moura foi realizada através de imagens de satélites onde se priorizou unidades com matas nativas. Nos municípios selecionados (Alta Floresta D’Oeste, Castanheira, Parecis e Rolim de Moura) foram escolhidas então 5 (cinco) unidades amostrais (UA) para a redução do espaço amostral e melhor avaliação do número de espécies com potencial oleígeno para produção de

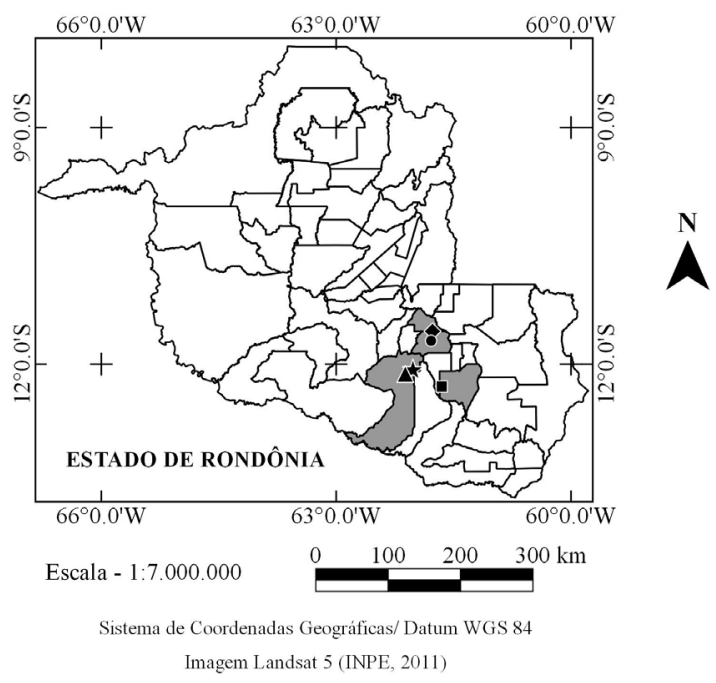

Figura 1. Seleção do espaço amostral: Mapa do Estado de Rondônia, com destaque à área de estudo; ( $\bullet$ Unidade Amostral 1 (UA1, coordenadas: S

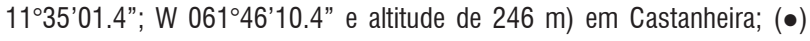
Unidade Amostral 2 (UA2, coordenadas: S 1142'14.6"; W 06147'16.4" e altitude de $260 \mathrm{~m}$ ) em Rolim de Moura; ( $\star$ ) Unidade Amostral 3 (UA3,

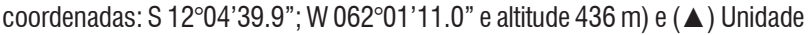
Amostral 4 (UA4, coordenadas: S 1207'27.7"; W 06207'00.0" e altitude 439 m) em Alta Floresta D' Oeste e (ロ) Unidade Amostral 5 (UA5, coordenadas: S $12^{\circ} 17^{\prime} 00,37^{\prime \prime}$; W 061³9'05,1" e altitude 451 m) em Parecis.

biodiesel. Na UA de Rolim de Moura e na UA de Castanheira há predominância de Floresta Ombrófila Aberta Submontana. Em Alta Floresta D'Oeste, as duas UA's selecionadas apresentam floresta predominante do tipo Ombrófila Densa e no município de Parecis, a UA selecionada apresenta vegetação predominante do tipo floresta Ombrófila aberta.

Em Rolim de Moura, Castanheira e Alta Floresta D'Oeste o tipo de solo predominante nas UA's selecionadas é o Latossolo Vermelho e em Parecis, a UA selecionada apresenta o solo do tipo areias quartzosas (Embrapa 2013). Esses solos apresentam-se como ácidos, com alto teor de alumínio e a consequente baixa fertilidade (Tabela 1).

A identificação das espécies foi realizada pelo método da Área Fixa (Moscovich et al. 1999) pois permite a seleção de árvores nas unidades amostrais proporcionais à área da unidade e à frequência dos indivíduos que nela ocorrem. Assim, cada unidade amostral foi demarcada selecionando-se um retângulo de $50 \mathrm{~m} \times 100 \mathrm{~m}$, totalizando $0,5 \mathrm{ha}$, onde as mesmas foram divididas em cinco faixas de $10 \mathrm{~m} \times 100 \mathrm{~m}$ e subdivididas em 10 subunidades de $10 \mathrm{~m} \times 10 \mathrm{~m}$.

Após a demarcação das unidades, os dados de campo foram inseridos em uma ficha contendo: número da árvore, Circunferência à Altura do Peito - CAP $(\geq 20 \mathrm{~cm})$ e identificação prévia com apoio técnico de funcionários da Secretaria Estadual do Meio Ambiente - SEDAM de Alta 
Tabela 1. Caracterização química de solos em fragmentos de Florestas de Rondônia na profundidade $0-20 \mathrm{~cm}$. UA1 = Unidade Amostral de Castanheira; UA2 = Unidade Amostral de Rolim de Moura; UA3 = Unidade Amostral de Alta Florestal; UA4 = Unidade Amostral de Alta Floresta; UA5 = Unidade Amostral de Parecis; $\mathrm{pH}=$ potencial Hidrogeniônico (1:1); $\mathrm{MO}=$ Matéria Orgânica $\left(\mathrm{g} \mathrm{kg}^{-1}\right) ; \mathrm{P}=$ Fósforo $\left(\mathrm{mg} \mathrm{kg}^{-1}\right) ; \mathrm{K}=$ Potássio $\left(\mathrm{mg} \mathrm{kg}^{-1}\right) ; \mathrm{Ca}+\mathrm{Mg}=\mathrm{Cálcio}+$ Magnésio $\left(\mathrm{cmolc} \mathrm{kg}^{-1}\right) ; \mathrm{Al}=$ Alumínio $\left(\mathrm{cmolc} \mathrm{kg}^{-1}\right) ; \mathrm{H}+\mathrm{Al}=$ Hidrogênio + Alumínio $\left(\mathrm{cmolc} \mathrm{kg}{ }^{-1}\right) ; \mathrm{CTC}=$ Capacidade de Troca de Cátions $\left(\mathrm{cmolc} \mathrm{kg}^{-1}\right) ; \mathrm{V}$ $=$ saturação de bases (\%).

\begin{tabular}{|c|c|c|c|c|c|}
\hline \multirow{2}{*}{ Atributos de Caracterização } & \multicolumn{5}{|c|}{ Unidades Amostrais } \\
\hline & UA1 & UA2 & UA3 & UA4 & UA5 \\
\hline $\mathrm{pH}_{\text {água }}$ & 5,20 & 4,80 & 5,80 & 5,40 & 5,90 \\
\hline MO & 12,80 & 17,87 & 9,66 & 13,04 & 18,60 \\
\hline$P_{\text {Mehlich-1 }}$ & 1,60 & 1,03 & 1,33 & 1,02 & 1,84 \\
\hline $\mathrm{K}_{\text {Menlich-1 }}$ & 114,00 & 40,00 & 23,00 & 35,00 & 23,00 \\
\hline $\mathrm{Ca}+\mathrm{Mg}$ & 1,84 & 1,44 & 1,81 & 2,20 & 0,26 \\
\hline $\mathrm{Al}$ & 0,30 & 0,20 & 0,20 & 0,30 & 0,25 \\
\hline $\mathrm{H}+\mathrm{Al}$ & 2,98 & 2,91 & 2,80 & 3,06 & 2,80 \\
\hline СTC & 5,10 & 4,44 & 4,67 & 5,35 & 3,12 \\
\hline V & 41,67 & 34,60 & 40,02 & 42,89 & 10,22 \\
\hline
\end{tabular}

Floresta D’Oeste - RO. O material botânico foi coletado para preparação de exsicatas de acordo com as técnicas usuais de herborização. O material herborizado foi identificado por especialistas da Coordenaçáo de Botânica do Museu Paraense Emílio Goeldi junto ao Herbário João Murça Pires (MG). Após a identificação das espécies, algumas amostras foram encaminhadas para o Herbário da Amazônia Meridional - HERBAM em Alta Floresta - MT e para a Universidade do Estado de Mato Grosso - UNEMAT. As famílias foram atualizadas de acordo com classificação adotada pelo APG III (2009) e os nomes científicos foram confirmados e atualizados pela Lista de Espécies da Flora do Brasil (Lista de Espécies da Flora do Brasil 2015).

Com os dados de campo foram calculadas a Frequência Absoluta (FA), que indica a porcentagem de parcelas que apresentam as espécies oleaginosas e a Frequência Relativa (FR), que é a razão da FA da espécie oleaginosa pela somatória das FAs de todas as espécies. Estes dados foram obtidos mediante uso das Equaçóes 1 e 2 para FA e FR, respectivamente:

$$
\begin{aligned}
& F A=\frac{\mathrm{ki}}{\mathrm{k}} \cdot 100 \\
& F R=\frac{\mathrm{FA}}{\sum F A} \cdot 100
\end{aligned}
$$

Onde: $\mathrm{FA}=$ Frequência Absoluta (\%); FR = Frequência Relativa (\%); ki = Número de subunidades onde ocorre a espécie em questão; $\mathrm{k}$ = Número total de subunidades.

\section{Coleta das oleaginosas, extrações e determinações químicas}

A coleta das oleaginosas (sementes e frutos em estágio maduro) foi realizada dentro das unidades amostrais. Quando não havia condições de coleta (ausência da espécie, número insuficiente de exemplares $(<3)$, espécies com CAP $<20$ centímetros ou sem frutos) foi realizada nas proximidades da unidade amostral selecionada. Ao total, foram coletados 15 quilogramas de cada uma das 10 espécies oleaginosas encontradas: Euterpe oleracea Mart. (açaí), Socratea exorrhiza (Mart.) H. Wendl. (palmeira barriguda), Iriartea deltoidea Ruiz \& Pav. (palmeira sete pernas), Attalea phalerata Mart. ex Spreng. (palmeira urucuri), Maximiliana maripa (Aubl.) Drude (inajá), Astrocaryum tucuma Mart. (tucumã-doAmazônas), Bertholletia excelsa Bonpl. (castanha do Brasil), Carapa guianensis Aubl. (andiroba), Orbignya phalerata Mart. (babaçu), Copaifera multijuga Hayne (copaíba). As análises de teor de lipídios e umidade das sementes e frutos coletados foram realizadas no laboratório de química da Universidade Federal de Rondônia.

O teor de umidade foi determinado pelo método da estufa (Sterilifer, SX CR/42, Piracicaba, SP, Brasil), por 24 horas, à temperatura de $105{ }^{\circ} \mathrm{C}$ (Brasil 2009). Já o teor de lipídios foi determinado na parte oleígena (endocarpo, mesocarpo ou epicarpo) dos frutos com extração por Soxhlet durante 24 horas. Como os lipídios têm uma grande faixa de hidrofobicidade, empregou-se o éter de petróleo (P.A, Exôdo), um solvente apolar (Martins et al. 2013), de acordo com o método proposto por AOCS (1998).

Com base no teor de lipídios foram selecionadas as espécies de maior teor oleígeno, sendo, portanto, realizada 
uma nova extração por solvente, com o mesmo aparato e solvente utilizado na determinação do teor de lipídeos, em volume suficiente para a produção de biodiesel $(-300 \mathrm{~mL})$. Depois da extração, os óleos foram colocados em evaporador rotativo (Quimis, Q344B1, Diadema, SP, Brasil), sob pressão reduzida, a cerca de $50^{\circ} \mathrm{C}$ para remover traços de solvente. Náo foi realizado processo de refino para esses óleos.

\section{Produção do biodiesel}

A produção de biodiesel prescindiu da análise do índice de acidez (ASTM 2001) nos óleos selecionados para a determinação da quantidade de catalisador hidróxido de sódio ( $\mathrm{NaOH}, \mathrm{P} . \mathrm{A}$, Exôdo) a ser utilizado na reação de transesterificação. Após a obtenção da massa de hidróxido de sódio, o biodiesel foi preparado em escala de bancada, de acordo com a metodologia proposta por Gonzalez et al. (2008). Para tal, utilizou-se cerca de $200 \mathrm{~mL}$ do óleo obtido anteriormente por extração com solvente, pressão atmosférica, temperatura de ebulição do álcool $\left(65^{\circ} \mathrm{C}\right.$, pois foi utilizado o metanol ( $\mathrm{CH}_{3} \mathrm{OH}$, Merck)), razão molar álcool:óleo de 6:1 e $1 \%$ do catalisador hidróxido de sódio (mais a massa devido à neutralização da acidez do óleo). As espécies utilizadas para a produção foram Carapa guianensis, Orbignya phalerata, Attalea phalerata, Copaifera multijuga, Bertholletia excelsa e Astrocaryum tucuma.

Para o cálculo da razão molar foram utilizadas informaçóes já disponíveis na literatura sobre a composição química dos óleos de Carapa guianensis (Silva 2005), Orbignya phalerata (Santos et al. 2007), Attalea phalerata (Moraes et al. 1996), Copaifera multijuga (Veiga Junior et al. 2007), Bertholletia excelsa (Costa et al. 2011) e Astrocaryum tucuma (Barbosa et al. 2009).

O sistema reacional foi mantido sob agitação, com posterior separaçáo da glicerina por simples decantaçáo e lavagem com água quente $\left(60^{\circ} \mathrm{C}\right)$. $\mathrm{O}$ processo de lavagem visou remover o excesso de álcool, sabóes, residuais de catalisador e de glicerina, restando apenas os ésteres formados juntamente com o óleo não convertido. O rendimento do biodiesel não foi avaliado e a taxa de conversão do óleo em biodiesel foi medida de hora em hora com base nos dados de RMN ${ }^{1} \mathrm{H}$ (Varian, Mercury 300, Palo Alto, Califórnia, USA), conforme metodologia proposta por Costa Neto et al. (2004). Esta metodologia consiste em inserir uma pequena quantidade $(50 \mu \mathrm{L})$ de amostra, previamente extraída com clorofórmio ( $\mathrm{CHCl}_{3}$ Quimex), num tubo de $\mathrm{RMN}{ }^{1} \mathrm{H}$ e avolumar para $650 \mu \mathrm{L}$ com clorofórmio deuterado $\left(\mathrm{CDCl}_{3}\right.$, Isotec), a uma temperatura controlada $\left(29^{\circ} \mathrm{C}\right)$. O tempo total gasto para o processo de conversão não ultrapassou 4 horas.

Para avaliar a qualidade do biodiesel obtido foram utilizados métodos cromatográficos seguindo as normas europeias EN 14103:2003, EN 14105:2003 e EN 14110:2003 para as análises de ésteres, glicerol (livre, total, monoglicerídeos, diglicerídeos e triglicerídeos) e metanol, respectivamente, de acordo com a Resolução n 45 de 25 de Agosto de 2014 da Agência Nacional do Petróleo (ANP 2014). Todos os ensaios foram realizados nos laboratórios Central Analítica e Central Analítica de Combustível da Universidade Federal de Mato Grosso, campus de Cuiabá, utilizando-se a técnica da cromatografia gasosa.

O teor de ésteres foi determinado em cromatógrafo (Shimadzu, GC-2010 com autoinjetor AOC5000, Kyoto Japão) equipado com detector de ionização em chama (DIC), injetor em modo split com razão de 1:50, com injeção de amostras líquidas $(1 \mu \mathrm{L})$ e software CGSolution para tratamento de dados. Foi utilizada uma coluna de polietilenoglicol (RestekStabilwax) com dimensóes de $30 \mathrm{~m}$, $0,25 \mathrm{~mm}$ de diâmetro interno e $0,25 \mu \mathrm{m}$ de espessura de filme com fluxo do gás de arraste $\left(\mathrm{H}_{2}\right)$ de $45 \mathrm{~cm} \mathrm{~s}^{-1}$. A temperatura do injetor e do detector foi de $250{ }^{\circ} \mathrm{C}$ e a da coluna $120{ }^{\circ} \mathrm{C}$ com permanência por 2 minutos, $10^{\circ} \mathrm{C}$ por minuto até 180 ${ }^{\circ} \mathrm{C}$ com permanência por 3 minutos, $5^{\circ} \mathrm{C}$ por minuto até $230{ }^{\circ} \mathrm{C}$ com permanência por 2 minutos. A quantificação foi realizada por comparação de área com o padrão interno heptadecanoato de metila (Sigma Aldrich, z 99\%).

As análises cromatográficas na determinação do metanol foram realizadas no mesmo cromatógrafo, detector e software para tratamento dos dados utilizado para a determinaçáo do teor de ésteres, agora em modo headspace, com injetor em modo split e razão de 1:50. O fluxo do gás de arraste $\left(\mathrm{H}_{2}\right)$ foi de $50 \mathrm{~cm} \mathrm{~s}^{-1}$ em uma coluna RestekRtx-1 (100\% dimetilpolisiloxano) com dimensóes de $30 \mathrm{~m}, 0,32 \mathrm{~mm}$ de diâmetro interno e $3 \mu \mathrm{m}$ de espessura de filme. A temperatura do injetor e do detector foi de $150{ }^{\circ} \mathrm{C}$, da coluna $50{ }^{\circ} \mathrm{C}$ isotérmica por 10 minutos e a temperatura de equilíbrio para amostragem por headspace foi de $80{ }^{\circ} \mathrm{C}$, com agitaçáo constante por 45 minutos. Após esse período o volume coletado e injetado foi de $500 \mu \mathrm{L}$ e a quantificação foi realizada utilizando curva analítica com calibração externa utilizando metanol grau HPLC ( J.T.Baker, $\geq 99,95 \%$ ).

As análises cromatográficas na determinação do teor de glicerídeos foram realizadas em um cromatógrafo a gás (Shimadzu, GC-2010 com autoinjetor AOC-20i, Kyoto, Japão), equipado com detector de ionização em chama (DIC). Foi utilizada uma coluna de DB5-HT com dimensóes de 15 $\mathrm{m}, 0,32 \mathrm{~mm}$ de diâmetro interno e $0,10 \mu \mathrm{m}$ de espessura de filme. Foi realizada injeção em modo direto utilizando-se gás de arraste $\left(\mathrm{H}_{2}\right)$ com pressão constante à $52 \mathrm{KPa}$. A temperatura inicial do injetor foi de $70{ }^{\circ} \mathrm{C}$ permanecendo por 1 minuto, taxa de $20^{\circ} \mathrm{C} \min ^{-1}$ até $380^{\circ} \mathrm{C}$ permanecendo nesta até o final da análise. A temperatura inicial do forno foi de $50^{\circ} \mathrm{C}$ por 1 minuto, taxa de $15^{\circ} \mathrm{C} \min ^{-1}$ até $180^{\circ} \mathrm{C}$, em seguida, taxa de 7 ${ }^{\circ} \mathrm{C} \min ^{-1}$ até $230{ }^{\circ} \mathrm{C}$, logo depois, taxa de $10^{\circ} \mathrm{C} \min ^{-1}$ até 380 
${ }^{\circ} \mathrm{C}$ permanecendo por 10 minutos. A temperatura do detector foi mantida constante a $380^{\circ} \mathrm{C}$ e a quantificação foi realizada utilizando curva analítica com padrão Individual Stock and Internal Standards Mix Kit (Sigma-Aldrich), sendo os padrōes internos do próprio kit.

\section{RESULTADOS}

Nas unidades amostrais selecionadas na regiáo de estudo foi possível encontrar as oleaginosas: Euterpe oleracea, na UA1 em Castanheira e Socratea exorrhiza, Iriartea deltoidea e Attalea phalerata nas UA3 de Alta Floresta e UA5 de Parecis (Tabela 2).

Apesar de haver exemplares de Maximiliana maripa, Astrocaryum tucuma, Bertholletia excelsa e Carapa guianensis na UA5, as mesmas foram coletadas em suas proximidades, pois dentro da unidade amostral o número de exemplares foi inferior a três ou encontravam-se inférteis.

Nas proximidades da UA3 foram obtidos os frutos de Orbignya phalerata, pois dentro desta unidade amostral não havia nenhum exemplar. Também foi coletado o óleo de Copaifera multijuga diretamente do único exemplar identificado na UA1. Esta espécie não consta no levantamento fitossociológico, pois ficou fora dos critérios de seleção (exemplar em número menor do que três).

A UA1 registrou o maior número de espécies (49) distribuídas em 28 famílias e 424 indivíduos; na UA2, 43 espécies, 22 famílias e 440 indivíduos; na UA3, 47 espécies, 23 famílias e 428 indivíduos amostrados; na UA4, 36 espécies, 21 famílias e 391 indivíduos e na UA5, 38 espécies, 22 famílias e 416 indivíduos, destes, 40 indivíduos oleígenos.

O Attalea phalerata, Orbignya phalerata e Astrocaryum tucuma, a Bertholletia excelsa a Carapa guianensis apresentaram os maiores teores de óleo (Tabela 3) e somente estas amostras foram utilizadas para a produção de biodiesel. Além destas espécies, foi inserido o óleo de Copaifera multijuga, que não foi determinado o teor de lipídios, pois o óleo foi extraído diretamente da árvore. A acidez apresentada por este óleo foi uma das mais elevadas, $12,64 \%$, exigindo assim uma maior massa de hidróxido de sódio para a reação de conversão do óleo em biodiesel (17,93 mg) (Tabela 4).

A reação de transesterificação foi monitorada por meio dos espectros do $\mathrm{RMN}{ }^{1} \mathrm{H}$ das amostras de óleo e os seus respectivos biodieseis formados. Nesta análise, o espectro do óleo (triacilglicerídeo) do Astrocaryum tucuma apresenta dois duplos dubletos relativo aos hidrogênios (em destaque) dos ésteres na regiáo entre 4,05 e 4,35 ppm (Figura $2 \mathrm{~A}$ ). A diminuição deste duplo dubleto na referida região e o aparecimento dos hidrogênios (em destaque) provenientes dos ésteres em outra região $(-3,65 \mathrm{ppm})$ é sinal que a reação de obtenção de biodiesel (transesterificaçáo) está ocorrendo (Figura 2 B). No presente estudo, o biodiesel foi preparado pela rota metílica e, portanto, o duplo dubleto do óleo desaparece e o éster metílico aparece. $\mathrm{O}$ monitoramento da conversão dos demais óleos seguiram esta mesma interpretação.

O óleo de Astrocaryum tucuma proporcionou um biodiesel com maior teor de ésteres (99,2\%), seguidos da Bertholletia excelsa $(98,8 \%)$, Orbignya phalerata $(98,3 \%)$ e Carapa guianensis $(84,6 \%)$. Para todos estes biodieseis formados, o residual de metanol foi igual a zero ( $0 \%$ em massa, ou seja, sem resíduo de álcool da reação), já os percentuais de glicerol (livre, total, monoglicerídeos, diglicerídeos e triglicerídeos), variaram dependendo do biodiesel da espécie em questão (Tabela 5).

\section{DISCUSSÃO}

$\mathrm{Na}$ UA1 verificou-se que Euterpe oleracea foi a única espécie oleaginosa com número de exemplares maiores do que três, em 0,5 ha (tamanho total da área) apresentou 5 (cinco) indivíduos, resultando em uma Frequência Absoluta de 8\% e Relativa de $1,5 \%$, o que significa que a presença desta espécie na comunidade é muito baixa.

Nas unidades amostrais 2 e 4 não foram identificadas espécies oleaginosas. As espécies de palmeiras se apresentam em pequenas quantidades em área de mata e maior número em pastagens, pois são naturalmente resistentes ao fogo quando atingem a fase adulta. Este fato provavelmente teve influência também na baixa frequência de Euterpe oleracea da UA1 (Shanley e Medina 2005; Teixeira e Carvalho 2007; Gehring et al. 2011). As características do solo nas unidades amostrais também podem ter influenciado a presença de determinadas espécies (Ruiz e Alencar 2004).

A maior presença de oleaginosas foi verificada na UA3, totalizando 103 indivíduos oleígenos, dentre as quais se destaca Iriartea deltoidea com 57 indivíduos dentro da unidade amostral, resultando em um FA e FR de $52 \%$ e $8,3 \%$, respectivamente. Além disso, houve a presença de Socratea exorrhiza com 34 indivíduos, FA de $46 \%$ e FR de $7,3 \%$ e Attalea phalerata com 12 indivíduos, FA de $24 \%$ e FR de $3,8 \%$.

A UA5 apresentou Euterpe oleracea com 30 indivíduos, FA de 38\% e FR de 7,0\% e Iriartea deltoidea com 10 indivíduos, FA de $16 \%$ e FR de $3,0 \%$, mostrando também a baixa incidência de oleaginosas na UA de Parecis.

A umidade apresentada pelas oleaginosas das unidades amostrais e regióes próximas a elas (Tabela 3) revelam que as espécies possuem diferentes percentuais, com mínimo de $0,5 \pm 0,01 \%$ na Socratea exorrhiza e máximo de 77,1 $\pm 0,6 \%$ no Astrocaryum tucuma. O teor de umidade pode favorecer o crescimento de microrganismos de degradaçáo do óleo, exigindo cuidados extras no armazenamento das sementes para a futura extração do óleo. 
Tabela 2. Parâmetros fitossociológicos das Unidades Amostrais com até 3 indivíduos de cada espécie demarcadas em fragmentos de Florestas de Rondônia. UA1 = Unidade Amostral de Castanheira; UA2 = Unidade Amostral de Rolim de Moura; UA3 = Unidade Amostral de Alta Florestal; UA4 = Unidade Amostral de Alta Floresta; UA5 = Unidade Amostral de Parecis; $\mathrm{NI}=$ Número de Indivíduos FA (\%) = Frequência Absoluta em porcentagem; FR (\%) = Frequência Relativa em porcentagem.

\begin{tabular}{|c|c|c|c|c|c|c|}
\hline Local & Família & Principais Espécies & Oleaginosa & $\mathrm{Nl}$ & FA (\%) & FR $(\%)$ \\
\hline \multirow{7}{*}{ UA1 } & Melastomataceae & Bellucia grossularioides (L.) Triana & Não & 40 & 48 & 8,8 \\
\hline & Moraceae & Pseudolmedia multinervis Mildbr. & Não & 40 & 52 & 9,5 \\
\hline & Lauraceae & Nectandra sp. & Não & 37 & 50 & 9,1 \\
\hline & Bignoniaceae & Jacaranda copaia (Aubl.) D. Don & Não & 34 & 36 & 6,6 \\
\hline & Arecaceae & Euterpe oleracea Mart. & Sim & 5 & 8 & 1,5 \\
\hline & & Outras & Não & 269 & 502 & 64,5 \\
\hline & & Total & - & 424 & 696 & 100,0 \\
\hline \multirow{7}{*}{ UA2 } & Lauraceae & Nectandra sp. & Não & 103 & 84 & 17,4 \\
\hline & Fabacaeae & Inga sp. & Não & 26 & 40 & 7,0 \\
\hline & Moraceae & Pseudolmedia multinervis Mildbr. & Não & 13 & 26 & 5,4 \\
\hline & Melastomataceae & Bellucia grossularioides (L.) Triana & Não & 11 & 20 & 4,1 \\
\hline & Fabaceae & Cedrelinga cateniformis Ducke (Ducke) & Não & 9 & 18 & 4,1 \\
\hline & & Outras & Não & 279 & 539 & 62,0 \\
\hline & & Total & - & 440 & 727 & 100,0 \\
\hline \multirow{7}{*}{ UA3 } & Arecaceae & Iriartea deltoidea Ruiz \& Pav. & Sim & 57 & 52 & 8,3 \\
\hline & Moraceae & Pseudolmedia multinervis Mildbr. & Não & 48 & 54 & 8,6 \\
\hline & Meliaceae & Guarea sp. & Não & 54 & 52 & 8,3 \\
\hline & Arecaceae & Socratea exorrhiza (Mart.) H. Wendl. & Sim & 34 & 46 & 7,3 \\
\hline & Arecaceae & Attalea phalerata Mart. ex Spreng. & Sim & 12 & 24 & 3,8 \\
\hline & & Outras & Não & 248 & 402 & 63,4 \\
\hline & & Total & - & 428 & 626 & 100,0 \\
\hline \multirow{7}{*}{ UA4 } & Malvaceae & Chorisia speciosa A. St.-Hil. & Não & 62 & 68 & 11,6 \\
\hline & Burseraceae & Protium heptaphyllum (Aubl.) Marchand & Não & 44 & 46 & 7,8 \\
\hline & Moraceae & Pseudolmedia multinervis Mildbr. & Não & 35 & 48 & 8,2 \\
\hline & Lauraceae & Nectandra sp. & Não & 27 & 44 & 7,5 \\
\hline & Arecaceae & Socratea exorrhiza (Mart.) H. Wendl. & Não & 21 & 38 & 6,5 \\
\hline & & Outras & Não & 202 & 342 & 58,4 \\
\hline & & Total & - & 391 & 586 & 100,0 \\
\hline \multirow{7}{*}{ UA5 } & Arecaceae & Oenocarpus bataua Mart. & Não & 85 & 48 & 8,9 \\
\hline & Annonaceae & Anona sp. & Não & 53 & 62 & 11,5 \\
\hline & Lauraceae & Nectandra sp. & Não & 50 & 50 & 9,3 \\
\hline & Arecaceae & Euterpe oleracea Mart. & Sim & 30 & 38 & 7,0 \\
\hline & Arecaceae & Iriartea deltoidea Ruiz \& Pav. & Sim & 10 & 16 & 3,0 \\
\hline & & Outras & Não & 188 & 326 & 60,3 \\
\hline & & Total & - & 416 & 540 & 100,0 \\
\hline
\end{tabular}

O teor de lipídeos da amostra de Maximiliana maripa $(2,3 \pm 0,1 \%)$ apresenta-se bem abaixo daqueles relatados por Rodrigues et al. (2010), igual a 35,5\%. A diferença encontrada deve estar relacionada às condiçóes experimentais, uma vez que o referido autor empregou uma mistura de solventes mais efetiva na extração dos lipídeos totais (clorofórmio e metanol) e à expressão dos resultados (valores em base seca).
O Astrocaryum tucuma apresentou um teor de lipídios de $21,5 \pm 2,0 \%$ (epicarpo + mesocarpo). Este valor foi inferior ao encontrado por Ferreira et al. (2008) (40,5\%) e superior ao valor de Santos et al. (2013) (11,8\%). Provavelmente essas diferenças estão condicionadas a parte do fruto analisada (mesocarpo) por Ferreira et al. (2008) e pelo menor tempo de extração (6h), o solvente éter etílico (baixa polaridade (Martins 
Tabela 3. Teor de umidade e teor de lipídios das oleaginosas estudadas. SD = Desvio padrão das amostras realizadas em triplicadas.

\begin{tabular}{|c|c|c|c|c|}
\hline \multirow[b]{2}{*}{ Família } & \multirow[b]{2}{*}{ Espécie } & \multirow[b]{2}{*}{$\begin{array}{l}\text { Umidade } \\
(\%) \pm S D\end{array}$} & \multicolumn{2}{|c|}{ Lipídeos } \\
\hline & & & $\begin{array}{l}\text { Presente estudo } \\
\quad(\%) \pm S D\end{array}$ & $\begin{array}{l}\text { Liter. } \\
(\%)\end{array}$ \\
\hline Arecaceae & Açaí (Euterpe oleracea Mart) / Endocarpo & $17,1 \pm 0,5$ & $3,4 \pm 0,8$ & $4^{(i)}$ \\
\hline Arecaceae & Urucuri (Attalea phalerata Mart. ex Spreng.) / Endocarpo & $17,2 \pm 0,5$ & $60,7 \pm 3,0$ & $59,8^{\text {(ii) }}$ \\
\hline Arecaceae & $\begin{array}{l}\text { Tucumã-do-Amazonas (Astrocaryum tucuma Mart.) / } \\
\text { Epicarpo + Mesocarpo }\end{array}$ & $77,1 \pm 0,6$ & $21,5 \pm 2,0$ & $11,8^{\text {(iii) }}$ \\
\hline Arecaceae & $\begin{array}{l}\text { Inajá (Maximiliana maripa (Aubl.) Drude)) / Epicarpo + } \\
\text { Mesocarpo }\end{array}$ & $60,8 \pm 0,4$ & $2,3 \pm 0,1$ & $35,5^{\text {(iv) }}$ \\
\hline Arecaceae & $\begin{array}{l}\text { Palmeira barriguda (Socratea exorrhiza (Mart.) H. } \\
\text { Wendl.) / Endocarpo }\end{array}$ & $0,5 \pm 0,01$ & $0,14 \pm 0,01$ & $0,15^{(v)}$ \\
\hline Arecaceae & $\begin{array}{l}\text { Palmeira sete pernas (Iriartea deltoidea Ruiz \& Pav.) / } \\
\text { Endocarpo }\end{array}$ & $1,8 \pm 0,01$ & $0,4 \pm 0,01$ & NE \\
\hline Lecythidaceae & $\begin{array}{l}\text { Castanha do Brasil (Bertholletia excelsa Bonpl.) / } \\
\text { Amêndoa }\end{array}$ & $9,5 \pm 0,5$ & $69,2 \pm 4,8$ & $72,5^{(v i)}$ \\
\hline Arecaceae & Babaçu (Orbignya phalerata Mart.) / Endocarpo & $13,3 \pm 0,5$ & $62,8 \pm 4,6$ & 66,0 (vii) \\
\hline Meliaceae & Andiroba (Carapa guianensis Aubl.) / Sementes & $10,0 \pm 1,0$ & $19,5 \pm 0,5$ & 2 a $30^{(i)}$ \\
\hline
\end{tabular}

NE: Não Encontrado dados na literatura nas condições de análise para esta espécie, (i) Shalen e Medina (2005), (ii) Moraes et al. (1996), (iii) Santos et al. (2013), (iv) Rodrigues et al. (2010), (v) Meyer (2012), (vi) Chunhieng et al. (2008), (vii) Brasil (2005).

Tabela 4. Teor de acidez das oleaginosas empregadas na produção de biodiesel.

\begin{tabular}{ccrc}
\hline \multirow{2}{*}{ Família } & Espécie & \multicolumn{2}{c}{ Acidez } \\
\cline { 3 - 4 } & & (\% ácido oleico) & (mg NaOH g-1 óleo) \\
\hline Meliaceae & Andiroba (Carapa guianensis Aubl.) & 16,84 & 23,90 \\
Arecaceae & Babaçu (Orbignya phalerata Mart.) & 0,21 & 0,29 \\
Arecaceae & Urucuri (Attalea phalerata Mart. ex Spreng.) & 6,21 & 8,81 \\
Fabaceae & Copaíba (Copaifera multijuga Hayne) & 12,64 & 17,93 \\
Lecythidaceae & Castanha do Brasil (Bertholletia excelsa Bonpl.) & 0,33 & 0,48 \\
Arecaceae & Tucumã-do-Amazonas (Astrocaryum tucuma Mart.) & 4,58 & 6,49 \\
\hline
\end{tabular}

et al. 2013)) e a parte do fruto analisada (mesocarpo) usados na extração da oleaginosa por Santos et al. (2013).

O teor de lipídios encontrado para a Bertholletia excelsa $(62,8 \pm 4,6 \%)$ está de acordo com Ferreira et al. (2006) (61\%) e Chunhieng et al. (2008) (72,5\%). Enquanto que o Orbignya phalerata quando utilizando etanol como solvente, apresentou o teor de lipídeos de 45\% (Pinto et al. 2012). Atribui-se este valor ao tipo do solvente utilizado (polar)(Martins et al. 2013) e aos cuidados de pré-análise do referido autor, uma vez que o mesmo relata que a umidade da oleaginosa pode influenciar na extração do lipídeo. Os demais teores de lipídios encontrados neste estudo apresentam-se dentro da faixa de valores encontrada na literatura.
Das espécies selecionadas para a produção de biodiesel, Carapa guianensis e Copaifera multijuga apresentaram os maiores índices de acidez. Caso esta alta acidez seja de natureza química do óleo e não de sua degradação, pode se tornar um agravante na obtenção do biocombustível, pois na reação de transesterificação as unidades ácidas resultam em sabóes (Gonzalez et al. 2008). O autor complementa que os óleos vegetais, especialmente os produzidos a partir de oleaginosas típicas do Norte e Nordeste do país, possuem elevada acidez natural. Isso provavelmente se deve às características dos solos da região e que pode ser acentuada devido a processos enzimáticos de deterioração, caso típico do dendê, cujos frutos devem ser processados no máximo 48 horas após a colheita. 


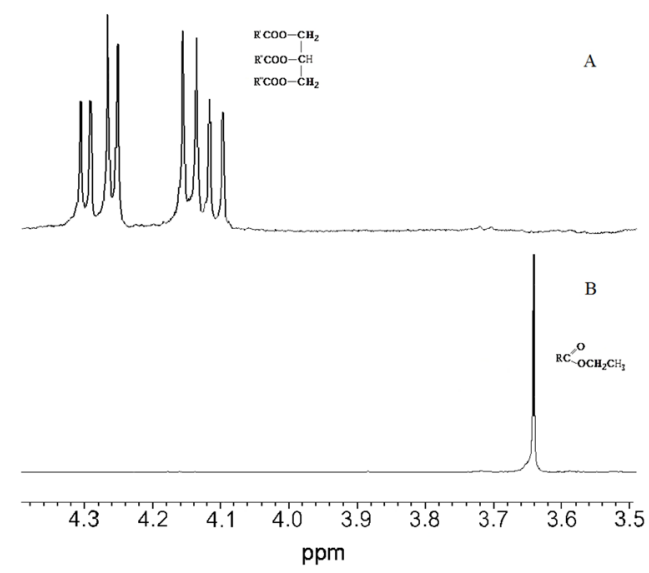

Figura 2. Análise de RMN ${ }^{1} \mathrm{H}$ para o monitoramento da conversão de óleo em biodiesel: (A) óleo do tucumã-do-Amazonas (Astrocaryum tucuma Mart.), (B) biodiesel do óleo do Astrocaryum tucuma.

Tabela 5. Certificação da qualidade do biodiesel obtido.

\begin{tabular}{|c|c|c|c|c|c|c|}
\hline \multirow{2}{*}{ Família } & \multirow{2}{*}{ Espécies } & \multicolumn{5}{|c|}{ Glicerol (\% e Massa) } \\
\hline & & Livre & Total & mono & $\mathrm{Di}$ & Tri \\
\hline Lecythidaceae & $\begin{array}{l}\text { Castanha do Brasil } \\
\text { (Bertholletia excelsa } \\
\text { Bonpl.) }\end{array}$ & 0,00 & 0,12 & 0,43 & 0,07 & 0,00 \\
\hline Arecaceae & $\begin{array}{l}\text { Tucumã-do-Amazonas } \\
\text { (Astrocaryum tucuma } \\
\text { Mart.) }\end{array}$ & 0,01 & 0,30 & 0,46 & 1,21 & 0,00 \\
\hline Meliaceae & $\begin{array}{l}\text { Andiroba } \\
\text { (Carapa guianensis } \\
\text { Aubl.) }\end{array}$ & 0,02 & 0,51 & 1,74 & 0,26 & 0,07 \\
\hline Arecaceae & $\begin{array}{l}\text { Babaçu } \\
\text { (Orbignya phalerata } \\
\text { Mart.) }\end{array}$ & 0,01 & 0,22 & 0,75 & 0,17 & 0,02 \\
\hline
\end{tabular}

O óleo de Copaifera multijuga não possibilitou a obtenção de biodiesel. Este fato deve ser consequente da natureza química do óleo tratar-se de sesquiterpenos e diterpenos ácidos (Tappin et al. 2004), que pelo processo da rota metílica clássica produziu sabão ao invés de biodiesel. O óleo de Carapa guianensis também apresenta ácidos em sua constituição (linoleico, esteárico, entre outros) (Ambrozin et al. 2006; Bataglion et al. 2014), mas a presença significativa de triacilglicerídeo possibilitou a obtenção do biodiesel.

Os espetros de acompanhamento da conversáo do óleo em biodiesel por RMN ${ }^{1} \mathrm{H}$ da palmeira Attalea phalerata indicam que houve a produçáo de biodiesel, entretanto, o produto da reação formada, após o desligar dos aparelhos que fizeram a conversão, apresentou-se posteriormente como um sólido e mesmo a temperatura superior a $70^{\circ} \mathrm{C}$ náo se liquefez. Por este motivo, não foi realizada análises adicionais nesse produto. Este fato provavelmente tem a ver com a natureza química do óleo, que ainda não foi completamente estudada. Apesar disso, esta oleaginosa nativa, com alto teor de óleo $(59,8 \%)$ (Moraes et al. 1996) foi apontada com potencial para a produção de biodiesel (Lima 2007; Negrelle 2013).

O teor de éster acima dos $98 \%$ aponta para a melhor conversão de óleo em biodiesel para a Bertholletia excelsa, Astrocaryum tucuma e Orbignya phalerata, já Carapa guianensis apresentou apenas $84,6 \%$ de ésteres. Isso já era esperado, pois existem poucas moléculas de triacilglicerídeos na Carapa guianensis para a conversão deste óleo em éster (Bataglion et al. 2014).

O teor de glicerol livre aponta para um possível resíduo de glicerina remanescente do processo de produçáo do biocombustível (Tabela 4). O valor máximo deste parâmetro é $0,02 \%$ para que náo provoque incrustaçóes no motor ciclo diesel (ANP 2014). Na indústria, por exemplo, altas concentraçóes de glicerol no biodiesel provocam problemas de armazenamento, pois quando o biodiesel é misturado com o diesel de petróleo, observa-se a separação do glicerol nos tanques de estocagem. Das oleaginosas utilizadas, Carapa guianensis mostrou o resultado mais elevado (0,02\%).

Os resultados mostraram que Carapa guianensis, Orbignya phalerata, Bertholletia excelsa e Astrocaryum tucuma têm potencial de produção do biocombustível. Especialmente para Astrocaryum tucuma, pois seus frutos já foram comercializados na regiâo para obtenção de óleo e demais subprodutos pela empresa INOVAM BRASIL, de Ji-Paraná - RO.

Do ponto de vista ambiental e ecológico, o extrativismo do Astrocaryum tucuma já foi viável no passado e garantia renda aos sitiantes. Atualmente, com a falta de compradores têm sido derrubadas nas pastagens, pois além de proporcionarem pouca sombra, seus espinhos são agressivos aos animais e seus frutos não são comestíveis pelo gado (Shanley e Medina, 2005). A espécie se desenvolve em solos pobres de terra firme, em áreas desmatadas, roçados, pastagens e capoeiras e sua boa resistência ao fogo o que garante a produçấo de frutos.

Neste sentido observa-se que existe maior potencial de produção de biodiesel a partir do Astrocaryum tucuma na região estudada, uma vez que resultou em um biodiesel de boa qualidade e sua coleta é livre, pois se trata de um produto florestal não madeireiro (Código florestal Brasileiro através da Lei ${ }^{\circ} 12.651$, de 25 de maio de 2012). Entretanto, como no estado de Rondônia não existe regulamentaçáo específica sobre os volumes e períodos de coleta do Astrocaryum tucuma, torna-se necessário um estudo da cadeia produtiva, assim como ocorre com a Carapa guianensis (Klimas et al. 2012), para que esta extração não descaracterize a cobertura vegetal existente e nem prejudique a função ambiental da área. Bem como no caso do pinhão manso (Spinelli et al. 2010; Rocha et al. 2012), a melhoria nos parâmetros genéticos desta espécie poderia potencializar o seu uso na região estudada. 


\section{CONCLUSÕES}

Os biocombustíveis obtidos das oleaginosas apresentaram-se adequados, com base nas análises realizadas, com exceção daquele obtido do óleo da Carapa guianensis (85\%), devido sua natureza ácida. O Astrocaryum tucuma destacou-se como a oleaginosa com maior potencial de produção de biodiesel pelo alto teor de lipídeos $(21,5 \%)$, pela possibilidade de obtenção de um biodiesel de qualidade (alta taxa de conversão) e por já ter existido na região um comércio de compra e venda deste fruto. Ressalta-se, portanto, a necessidade de um plano de utilização sustentável desta espécie.

\section{AGRADECIMENTOS}

Os autores agradecem ao CNPq pelo apoio financeiro, aos acadêmicos voluntários dos cursos de Engenharia Florestal e Agronomia; engenheiros florestais da SEDAM de Alta Floresta D’Oeste; Museu Paraense Emílio Goeldi/Coordenação de Botânica; Herbário da Amazônia Meridional; Proprietários das fazendas onde foram implantadas as unidades amostrais; Laboratório Central de Análise de Combustíveis e Laboratório Central Analítica da Universidade Federal do Mato Grosso, campus de Cuiabá - MT.

\section{BIBLIOGRAFIA CITADA}

Ambrozin, A.R.P.; Leite, A.C.; Bueno, F.C.; Vieira, P.C.; Fernandes, J.B.; Bueno, O. C., et al. 2006. Limonoids from andiroba oil and Cedrela fissilis and their insecticidal activity. Journal of the Brazilian Chemical Society, 17: 542-547.

ANP - Agência Nacional do Petróleo. 2014. Resolução ANP 45/2014. Disponível em http://nxt.anp.gov.br/nxt/gateway.dll. Acesso em 11/02/2015.

AOCS - American Oil Chemists Society. Official methods and recommended practices of the AOCS. Champaign: A.O.C.S., 1998.

APG III. 2009. An update of the Angiosperm Phylogeny Group classification for the orders and families of flowering plants: APG III. Botanical Journal of the Linnean Society, 161:105-121.

ASTM D664 - $11^{\circ}$ Standard Test Method for Acid Number of Petroleum Products by Potentiometric Titration (Reference Method), 2001.

Barbosa, B.S.; Koolen, H.H.F.; Barreto, A.C.; Silva, J.D.; Figliuolo, R.; Nunomura, S.M. 2009. Aproveitamento do óleo das amêndoas de tucumã do amazonas na produção de biodiesel. Acta Amazonica, 39: 371-376.

Bataglion, G.A.; Silva, F.M.A.; Santos, J.M.; Santos, F.N.; Barcia, M.T.; Lourenço, C.C. et al. 2014. Comprehensive characterization of lipids from Amazonian vegetable oils by mass spectrometry techniques. Food Research International, 64: 472-481.

Brasil. 2005. Ministério da Agricultura, Pecuária e Abastecimento. Anuário estatístico da agroenergia. Brasília. 2005, 120p.

Brasil. 2009. Ministério da Agricultura, Pecuária e Abastecimento. Regras para análise de sementes. Brasília. 2009, 399 p.
Chunhieng, T.; Hafidi, A.; Pioch, D.; Brochier, J.; Montet, D. 2008. Detailed study of Brazil nut (Bertholletia excelsa Bonpl.) oil micro-compounds: Phospholipids, tocopherols and sterols. Journal of the Brazilian Chemical Society, 19: 1374-1380.

Costa, P.A.; Ballus, C.A.; Teixeira Filho, J.; Godoy, H.T. 2011. Fatty acids profie of pulp and nuts of Brazilian fruits. Ciência e Tecnologia de Alimentos, 31: 950-954.

Costa Neto, P.R.; Caro, M.S.B.; Mazzuco, L.M.; Nascimento, M.G. 2004. Quantification of soybean oil ethanolysis with 1H NMR. Journal of the American Oil Chemists' Society, 81: 1111-1114.

Embrapa. 2013. Sistema brasileiro de classificação de solos. Centro Nacional de Pesquisa de Solos. 3. ed. revisada e ampliada. Brasília, 2013. 353p.

Ferreira, E.S.; Silveira, C.S.; Lucien, V.G.; Amaral, A.S. 2006. Caracterização físico-química da amêndoa, torta e composição dos ácidos graxos majoritários do óleo bruto da castanhado-brasil (Bertholletia excelsa Bonpl.). Alimentos e Nutrição Araraquara, 17: 203-208.

Ferreira, E.S.; Silveira, C.S.; Lucien, V.G.; Amaral, A.S.; Silveira, C.S. 2008. Caracterização físico-química do fruto e do óleo extraído de tucumã (Astrocaryum vulgare mart). Alimentos e Nutrição Araraquara, 19: 427-433.

Gehring, C.; Zelarayan, M.L.C.; Almeida, R.B.; Moraes, F.H.R. 2011. Allometria da palmeira babaçu em um agroecossistema de derruba-e-queima na periferia este da Amazônia. Acta Amazonica, 41: 127-134.

Gonzalez, W.A.; Machado, C.R.; Mendonça, N.B.; Borges, L.E.P. 2008. Tecnologia Para Aproveitamento da Cadeia Produtiva de Dendê. Gonzales, W.A.; Machado, C.R.; Barreto, E.J.F.; Dall'Oglio, E.L.; Correia, J.C. et al. (Ed.). Biodiesel e Óleo Vegetal in Natura: Soluçōes Energéticas para a Amazônia. v.1. Ministério de Minas e Energia, Brasília, Distrito Federal, p. 78-97.

Iha, O.K.; Alves, F.C.S.C.; Suarez, P.A.Z.; Silva, C.R.P.; Meneghettib, M.R.; Meneghettib, S.M.P. 2014. Potential application of Terminalia catappa L. and Carapa guianensis Aubl. oils for biofuel production: Physical-chemical properties of neat vegetable oils, their methyl-esters and bio-oils (hydrocarbons). Industrial Crops and Products, 52: 95-98.

Klimas, C.A.; Kainer, K.A.; Wadt, L.H.O. 2012. The economic value of sustainable seed and timber harvests of multi-use species: An example using Carapa guianensis Aubl. Forest Ecology and Management, 268: 81-91.

Lima, J.M.T. 2007. Florescimento e frutificação em duas palmeiras oleaginosas do gênero Attalea nos estados do Acre e Rondônia, Brasil: uma fonte potencial de óleo para biodiesel. Revista Brasileira de Agroecologia, 2: 1310-1313.

Lista de Espécies da Flora do Brasil. Jardim Botânico do Rio de Janeiro. Disponível em: <http://floradobrasil.jbrj.gov.br/>. Acesso em: 16 Mar. 2015.

Martins, C.R.; Lopes, W.A.; Andrade, J.B. 2013. Solubilidade das substâncias orgânicas. Quimica Nova, 36: 1248-1255.

Meyer, J.M. 2012. Teor e composiçāo de ácidos graxos de óleos de frutos de palmeiras nativas. Dissertação de mestrado, Instituto 
de Biociências / Universidade de São Paulo, São Paulo, São Paulo. 90p.

Moraes R.M.; Borchsenius, F.; Blicher-Mathiesen, U. 1996. Notes on the biology and uses of the motacú palm (Attalea phalerata Mart. ex. Spreng., Arecaceae) from Bolivia. Economic Botany, 50: 423-428.

Moscovich, F.A.; Brena, A.B.; Longhi, S.J. 1999. Comparação de diferentes métodos de amostragem, de área fixa e variável, em uma floresta de Araucaria angustifólia (Bertol.) Kuntze. Ciência Florestal, 9: 173-191.

Negrelle, R.R.B. 2013. Estrutura populacional e potencial de regeneração de Attalea phalerata Mart. ex Spreng. (acuri). Ciência Florestal, 23: 727-734.

Pinto, L.F.; Silva, D.I.S.; Silva, F.R.; Santos, V.B.; Soletti, J.I.; Carvalho, S.H.V. 2012. Assessment of the feasilibility of different oil sources to biodiesel production. Acta Scientiarum. Technology, 34: 227-231.

Ramos, L.P.; Domingos, A.K.; Kucek, K.T.; Wilhelm, H.M. 2003. Biodiesel: Um projeto de sustentabilidade econômica e sócioambiental para o Brasil. Biotecnologia: Ciência e Desenvolvimento, 31: 28-37.

Ramos, L.P.; Wilhelm, H.M. 2005. Current status of biodiesel development in Brazil. Applied Biochemistry and Biotechnology, 121-124: 807-820.

Rocha, R.B.; Ramalho, A.R.; Teixeira, A.L.; Laviola, B.G.; Silva, F.C.G.; Militão, J.S.LT. 2012. Eficiência da seleção para incremento do teor de óleo do pinhão-manso. Pesquisa Agropecuária Brasileira, 47: 44-50.

Rodrigues, A.M.C.; Darnet, S.; Silva, L.H.M. 2010. Fatty Acid profiles tocopherol of buriti (Mauritia flexuosa L.f.), patawa (Oenocarpus bataua Mart.), tucumã (Astrocaryum vulgare Mart.), mari (Poraqueiba paraensis Ducke) and inajá (Maximiliana maripa (Aubl.) Drude) fruits. Journal of the Brazilian Chemical Society, 21: 2000-2004.

Ruiz, R.R.; Alencar, J.C. 2004. Comportamento fenológico da palmeira patauá (Oenocarpus bataua Mart.) na reserva florestal Adolpho Ducke, Manaus, Amazonas, Brasil. Acta Amazonica, 34: 553-558.
Santos, M.F.G.; Marmesat, S.; Brito, E.S.; Alves, R.E.; Dobarganes, M.C. 2013. Major components in oils obtained from Amazonian palm fruits. Grasas y Aceites, 64: 531-536.

Santos, N.A.; Tavares, M.L.A.; Rosenhaim, R.; Silva, F.C.; Fernandes Jr., V.J.; Santos, I.M.G.; Souza, A.G. 2007. Themogravimetric and calorimetric evaluation of babassu biodiesel obtained by the methanol route. Journal of Thermal Analysis and Calorimetry, 87: 649-52.

Shanley, P.; Medina, G. 2005. Frutiferas e Plantas Úteis na Vida Amazônica. Belém: CIFOR, Imazon, 2005.

Silva, C.L.M. 2005. Obtenção de ésteres etílicos a partir da transesterificação do óleo de andiroba com etanol. Dissertação de mestrado, Instituto de Química/ Universidade Estadual de Campinas, Sáo Paulo. 64p.

Spinelli, V.M.; Rocha, R.B.; Ramalho, A.R.; Marcolan, A.L.; Vieira Jr, J.R.; Fernandes, C.F.; Militão, J.S.L.T.; Dias, L.A.S. 2010. Componentes primários e secundários do rendimento de óleo de pinhão-manso. Ciência Rural, 40: 1752-1758.

Tappin, M.R.R.; Pereira, J.F.G.; Lima, L.A.; Siani, A.C.; Mazzei, J.L.; Ramos, M.F.S. 2004. Análise química quantitativa para a padronização do óleo de copaíba por cromatografia em fase gasosa de alta resolução. Química Nova, 27: 236-240.

Teixeira, M.A.; Carvalho, M.G. 2007. Regulatory mechanism for biomass renewable energy in Brazil, a case study of the Brazilian babassu oil extraction industry. Energy, 32: 999-1005.

Veiga Junior, V.F.; Rosas, E.C.; Carvalho, M.V.; Henriques, M.G.M.O.; Pinto, A.C. 2007. Chemical composition and anti-inflammatory activity of copaiba oils from Copaifera cearensis Huber ex Ducke, Copaifera reticulata Ducke and Copaifera multijuga Hayne - A comparative study. Journal of Ethnopharmacology, 112: 248-254.

Villela, A.A.; Jaccoud, D.B.; Rosa, L.P.; Freitas, M.V. 2014. Status and prospects of oil palm in the Brazilian Amazon. Biomass Bioenergy, 67: 270-278.

Recebido em 02/04/2015

Aceito em 08/06/2015 